\title{
Crisis internacionales y economías emergentes de la alianza del pacífico: escenarios y análisis
}

\author{
Pablo Rijalba Palacios ${ }^{1}$ \\ prijalbap@unp.edu.pe \\ https://orcid.org/0000-0002-4730-105X \\ Wilmer Eduardo Litano Boza ${ }^{2}$ \\ wlitanob@unp.edu.pe \\ https://orcid.org/0000-0003-1289-1552 \\ Universidad Nacional de Piura
}

\section{RESUMEN}

Se analiza la relación entre escenarios de crisis y sus efectos en mercados financieros emergentes de la Alianza del Pacífico. Estas economías latinoamericanas muestran sistemas cambiarios flexibles y están conectados con significativa apertura económica y financiera. Se proponen escenarios de crisis de carácter global las cuales manifiestan sus efectos en los mercados reales y financieros. Se postula que, cualesquiera sean las causas de una crisis, una vez manifestada y posicionada se traslada hacia el resto de la economía vía inductores hacia los mercados y financieros. Para tal fin, se explora ¿Cuáles son los indicadores y señales que están presentes antes, durante y después de la ocurrencia de choques de alcance mundial? Se aplica metodología basada en sistematización de evidencias, estado del arte y teorías de crisis, análisis estadístico y econométrico como marco para entender la conexión de indicadores y señales presentes en los mecanismos de transmisión de crisis, específicamente en países de la Alianza del Pacífico: México, Colombia, Chile y Perú. Se postula también que, la presencia de crisis muestra diferencias y similitudes, como ha sucedido durante la crisis suprime, como estaría sucediendo en el actual escenario de emergencia sanitaria por COVID 19; y podría suceder como consecuencia de la vaticinada crisis de recalentamiento para el 2023.

Palabras claves: crisis internacional; países emergentes; alianza del pacífico

\footnotetext{
${ }^{1}$ Catedrático de la Facultad de Economía, Programas de Maestría de Escuela de Post Grado y doctorando del Programa de Doctorado en Ciencias Económicas y Financieras de la Universidad Nacional de Piura.

${ }^{2}$ Catedrático de la Facultad de Economía y docente del Programa de Doctorado en Ciencias Económicas y Financieras de la Universidad Nacional de Piura.
} 


\title{
International crises and emerging economies of the pacific alliance: scenarios and analysis
}

\begin{abstract}
ABSTRAC
The relationship between crisis scenarios and their effects on emerging financial markets of the Pacific Alliance is analyzed. These Latin American economies show flexible exchange rate systems and are connected with significant economic and financial openness. Global crisis scenarios are proposed which manifest their effects on the real and financial markets. It is postulated that, whatever the causes of a crisis, once manifested and positioned, it is transferred to the rest of the economy via inducers to markets and financials. To this end, it explores What are the indicators and signals that are present before, during and after the occurrence of global shocks? Methodology based on the systematization of evidence, state of the art and crisis theories, statistical and econometric analysis is applied as a framework to understand the connection of indicators and signals present in the mechanisms of crisis transmission, specifically in countries of the Pacific Alliance: Mexico, Colombia, Chile and Peru. It is also postulated that the presence of crisis shows differences and similarities, as has happened during the crisis subprime, as would be happening in the current health emergency scenario due to COVID 19; And it could happen as a consequence of the predicted overheating crisis for 2023.
\end{abstract}

Keywords: international crisis; emerging markets; pacific alliance

Artículo recibido: 10 Setiembre. 2021 Aceptado para publicación: 15 Octubre. 2021

Correspondencia: prijalbap@unp.edu.pe Conflictos de Interés: Ninguna que declarar 


\section{INTRODUCCIÓN}

La realidad problemática se sustenta en la evidencia mostrada desde el siglo XX, la cual ha mostrado la ocurrencia de diferentes crisis financieras con repercusiones en todo el sistema internacional (Odono, CN; 2000). Estas crisis normalmente están asociadas a factores como bajas tasas de interés y, a impulsos de los inversionistas en buscar mejores retornos en el exterior. Estos factores contribuyeron a que, los antes llamados países del tercer mundo o economías en desarrollo, ahora sean reconocidos como mercados emergentes; y considerados como nueva frontera de oportunidades financieras (Odono, CN 2020). Bajo esta nueva categoría se incluyeron países dísimiles que vendían deuda y bonos; tal es caso de Polonia, Rusia, Turquia, Brasil, Indonesia, Tailandia, Venezuela, México, Filipinas, Malasia, Ecuador, Perú, Chile y Argentina, entre otros (Odono CN; 2000).

A partir de la denominada crisis subprime, se han manifestado tensiones financieras a causa de la creciente interdependencia global, lo cual ha acentuado la exposición a eventos predecibles y no predecibles, económicos y financieros; siempre de alcance internacional. Entre el 2007-2011, las economías emergentes se han visto sometidas a los efectos de las tensiones comerciales entre China-Estados Unidos, vía combinación de políticas fiscales y monetarias que propician la apreciación del dólar y endurecimiento de las condiciones financieras internacionales. En este contexto de turbulencia, en el año 2011 se constituye la Alianza del Pacífico, AP, conformada por cuatro países latinoamericanos -Colombia, Chile, México y Perú- con el fin de vincular el aspecto geoestratégico con la posibilidad de convertirse en puente con el Foro de Cooperación Asia Pacífico; y, medio de reacomodación de los Estados Unidos en América Latina (Ardila M, 2015).

Desde entonces, los deterioros en los indicadores financieros de economías de las AP no han sido homogéneos. Los actores económicos, en especial los inversores han estado ligados a turbulencias y condiciones financieras que se han manifestado, según Ardila $\mathrm{M}$ (2015) en indicadores habitualmente referidos a la apertura comercial, el tipo de cambio, las reservas internacionales netas, RIN, la deuda externa y la inversión extranjera directa, IED (Soto E R y Correa E; 2008); que de acuerdo con Landa H.O y Barron S (2021), agrega la tasa de interés, el ahorro interno y el impulso crediticio interno hacia empresas gobierno y familia. De manera similar, la presencia de choques se ve acentuada o 
amortiguada por la situación fiscal de cada país, reflejada principalmente en la deuda total y el déficit fiscal (Steinberg F, 2008).

Por otro lado, autores como Rubini (2010) y Ardila M (2015), consideran que la presencia de riesgo e incertidumbre, condicionan la forma como los mercados bursátiles se manifiestan ante el surgimiento de choques. Este recorrido lleva a preguntarnos si la actual crisis por emergencia sanitaria (COVID 19), ha revelado también estas vulnerabilidades, toda vez que, en base a lo señalado por Martín-Fiorino y Reyes G (2020), lo que empezó como shock de oferta se trasladó, rápidamente, hacia los mercados financieros acentuando la contracción de la demanda y el producto bruto interno, PBI. Recientemente, el Banco Central de la Federación Rusa, CBR, y la Revista Sputnik (2021), proyectan para el año 2023, una crisis sin precedentes que ya se estaría mostrando en señales de recalentamiento de las economías, inflación y deterioro de los sistemas financieros mundiales. Crisis, a la que los países de la Alianza Pacífico no escaparían una vez manifestada.

Es así como, la realidad problemática señala que, ante la presencia de escenarios de crisis de impacto global, cualesquiera sean sus causas, una vez manifestadas influyen en el comportamiento real y financiero; y que los mecanismos de transmisión revelan señales que adquieren particularidades en los mercados emergentes como sería el caso de los países de la AP. Estos, no sólo poseen mercados financieros sensibles, sino que además muestran similitudes en cuanto a su grado de apertura, sistemas cambiarios flexibles y respuestas ante vulnerabilidades y choques.

Bajo esta mirada nos debemos preguntar: ¿De qué manera ocurren los mecanismos de transmisión de las crisis internacionales hacia las economías emergentes de la Alianza del Pacífico considerando sus sensibilidades, señales y efectos en los sectores real y financiero? Para abordar este cuestionamiento, hay que reconocer los mecanismos por los cuales los mercados financieros de estos países se muestran sensibles ante escenarios de crisis, los cuales guardan relación, pero muestran diferencias. Nos referimos al fenómeno suprime, la emergencia sanitaria COVID 19 y la crisis financiera mundial por recalentamiento vaticinada por el CBR para el año 2023.

Como se aprecia, se trata de un tema de gran relevancia y justificación, pues se aborda escenarios de crisis mundiales que según Munchau W (2009), y como se postula en el presente artículo, las crisis no siempre son el resultado de procesos de liberalización 
financiera, burbujas suprime y/o excesos de liquidez global, también existen factores sistémicos, crisis de oferta; y, en el contexto actual, la crisis sanitaria que colocan al mundo al borde de la recesión. La evidencia señala que, al parecer, toda forma de crisis, una vez manifestada se traslada hacia los diferentes mecanismos y segmentos de los mercados; por lo que, el contexto debe ser analizado en términos de la alta sensibilidad de los mercados reales y financieros, los cuales con su accionar trasladan las turbulencias a los diferentes segmentos y agentes económicos.

El estado del arte del tema señala fuertes y claras conexiones entre variables financieras, reales y especulativas en escenarios de crisis. Al respecto, Torres (2012), manifiesta que una crisis financiera no es sino una perturbación más o menos repentina que produce pérdidas en los activos financieros que tienen influencia decisiva en la marcha de los negocios y la actividad económica general. Duican y Pop (2015), analizan la relación entre flujos crediticios y crecimiento económico en Rumanía encontrando que los créditos otorgados por el sistema financiero afectan al crecimiento y desarrollo económico. Esto, respalda la dependencia entre la financiación que ofrecen los sistemas bancarios; y más aún cuando se ven acentuados por su expansión y la entrada de capitales extranjeros. Además, los resultados sugieren que las economías emergentes deben reafirmar sus políticas de financiamiento empresarial vía créditos por su significativa contribución en el crecimiento.

Por ello, es importante preguntarnos también ¿Qué sucedería en una economía, si se observara declinación del crédito durante la ocurrencia crisis financiera? ¿Afectaría, realmente, ello a la actividad económica? Y, de ser así, ¿cómo se recuperaría una economía sin créditos? Autores como Biggs, Mayer y Pick (2009), sugieren que el crecimiento del crédito y el impulso crediticio, siguen muy de cerca los movimientos que muestra la tasa de crecimiento del producto, tanto en fases contractivas como en fases de recuperación. Lo anterior, quiere decir que, el crecimiento del PBI está relacionado con cambios en el flujo de crédito más que los stocks o niveles de este. Es decir, el impulso crediticio -cambios en el flujo del crédito-, es particularmente relevante durante y después de recesiones.

La relación IED-crecimiento, muestra fuerte conexión. Autores como James Ang (2007), examinan esta relación en economías pequeñas y abiertas como Malasia, a través del control del nivel de desarrollo financiero. Los resultados muestran que la IED y el 
desarrollo financiero están relacionados positivamente con cambios del crecimiento a largo plazo. Esto quiere decir, que la IED estimula el crecimiento a través del desarrollo financiero, pero si el alcance y la eficiencia del sistema financiero de una economía no es suficiente, no se observarán efectos positivos de la IED.

En un estudio para siete países desarrollados (Estados Unidos, Japón, Alemania, Gran Bretaña, Italia, Francia y los Países Bajos) y para un largo período (1950-2001), Fink, Haiss y Hristoforova (2006), analizaron la relación entre PBI real y variables relacionadas al comportamiento de bonos, crédito y acciones. Los hallazgos mostraron patrones de causalidad que difieren debido a la heterogeneidad del mercado, la estructura y el diferente grado de apertura e integración internacional de los mercados de capitales en la muestra de país.

La evidencia a nivel de América Latina, AL, contribuye a entender que los flujos financieros -en cualquiera de sus formas- guardan relación con los procesos de crecimiento económico. Así, Alestre (2014), encuentra que para países miembros de la Organización para la Cooperación y el Desarrollo Económicos (OCDE), muestran ingreso alto y en los países miembros de la Eurozona muestran mayor proporción de crédito interno respecto al PIB, en relación con aquellos países más pobres -de ingreso medio y bajo-; situación que muestra también particularidades como el hecho de estar más endeudados. Según los autores estas diferencias se explican por los niveles de riesgo, problemas institucionales, ruido político, gobierno, factores macroeconómicos y jurídicos que condicionan este comportamiento.

Los autores señalan que, en América Latina, en los años '60 ocurrieron aumentos del crédito interno, pero caídas significativas en los años 90 provocada por el descenso brusco de esta variable en Brasil y Chile; encontrando, además que, en países como Brasil, Chile y Colombia, en donde se otorga más crédito interno (Brasil, Chile y Colombia) la correlación con el crecimiento del PIB es más fuerte; resultados que, según los autores, respaldan la tesis de Schumpeter. Estas relaciones se reafirman con los hallazgos de Tercero y Guercio (2011), cuando analizan la relación entre crecimiento económico y comportamiento de los sistemas financieros. Para tal fin, incluye PBI, capitalización bursátil, bonos, créditos y activos bancarios. Este hallazgo, permite afirmar que la estabilidad financiera es, entonces, fundamental, para garantizar el correcto funcionamiento del sistema financiero durante escenarios de crisis, en la medida que evita 
o suaviza las consecuencias de su propagación. Se observa que, en Brasil, Chile, Colombia, México y Perú, las correlaciones entre sistema financiero y PBI son elevadas. Lahura y Vargas (2013), abordan la relación entre sistema bancario y nivel de actividad, encontrando que la relación dinámica entre actividad e indicadores convencionales de intermediación financiera se observa considerando el uso de cuasidinero/PBI, liquidez/PBI y crédito/PBI. Los efectos de los choques deben ser diferenciados entre permanentes y transitorios, para el sistema bancario se explican más por choques transitorios.

Las crisis financieras internacionales han mostrado que entre los sectores real y financiero existe una estrecha relación, siendo importante que el sistema financiero observe buen funcionamiento para que la actividad económica funcione correctamente. No obstante, la evidencia también señala que, en el largo plazo, el impulso crediticio determina la evolución del sistema bancario y el PBI real. Sobre este punto, Lahura \& Vega (2011), determinaron que, a pesar de la inestabilidad el impulso crediticio (tasa de crecimiento del crédito), hasta el año 2010, en el Perú, el crédito no ha sido incluido en los modelos de proyección de elaboración de políticas monetarias, debido a la preponderancia de los precios relativos en dichos modelos.

Por otro lado, autores como Eiteman, Stonehill y Moffett (2011) señalan que el entorno financiero global expone a las economías emergentes a riesgos cambiarios que afectan a las decisiones de inversiones internacionales. Este comportamiento, además, interactúan con variables y escenarios bursátiles y los créditos internacionales. Al respecto, Jeff Madina (2016) la relación entre el comportamiento de los mercados financieros y el entorno global, los cuales condicionan las políticas financieras al interior de los países globalmente conectados. A partir de esta revisión se puede reflexionar acerca de ¿Cuáles son las principales señales previas a una crisis financiera internacional? ¿Qué teorías sustentan las crisis internacionales? ¿Cómo son y cómo ocurren los mecanismos de trasmisión de crisis hacia economías emergentes, considerando sus sensibilidades, desajustes, y efectos al interior de los países de la AP?

Además, interrogarse ¿Cuáles son los escenarios de crisis internacionales que pueden enfrentar las economías emergentes, en especial los de la AP, desde una perspectiva de choques? y ¿Cuáles son las diferencias y similitudes entre escenarios, reciente-presente- 
futuro, de crisis internacionales, como la suprime, la actual emergencia sanitaria por COVID 19 y el recalentamiento global proyectado por Rusia?

Finalmente, se reflexiona acerca de si, las crisis son predecibles o sólo tenemos señales y escenarios posibles; de ser así, también podemos analizar las razones por las cuales no existe -ni podría existir- una lista de recetas infalibles para la detectar crisis financieras; y más aún de carácter global. La cuestión es inferir lecciones y recomendaciones en base a la evidencia, y de manera particular en países emergentes que asumen pactos de colaboración como es el caso de los países de la Alianza del Pacífico.

\section{OBJETIVOS DE INVESTIGACIÓN}

El objetivo es analizar la relación y mecanismos de trasmisión de crisis internacionales hacia economías emergentes de la AP. Los objetivos específicos refieren a:

a. Reconocer las teorías de crisis financieras y los límites en su capacidad explicativapredictiva para economías emergentes.

b. Identificar señales recurrentes de crisis financieras que enfrentan las economías emergentes de los países de la AP.

c. Evidenciar cuáles serían los inductores de crisis internacionales dadas las condiciones emergentes de las economías de la AP.

d. Comparar escenarios, reciente-presente-futuro, de crisis incidiendo en la suprime, la emergencia sanitaria (COVID 19) y recalentamiento predicho por el CBR.

\section{MÉTODOS}

La propuesta metodológica se ubica en un nivel descriptivo, correlacional y explicativo. Para tal fin, la sistematización, el análisis de contenidos, la estructura base de datos, y la realización del análisis según la estadística descriptiva e inferencial a partir de relaciones causales señaladas por la teoría. Se plantea un modelo de panel data, y se explora evidencia respecto a si la presencia de crisis internacional, una vez generada, se manifiesta a través de mecanismos, señales e indicadores en el sector real y financiero. El diseño metodológico se basa en cuatro momentos (Tabla 1). 
Tabla 1: Momentos del diseño metodológico.

\begin{tabular}{ll}
\hline $\mathbf{N}^{\circ}$ & \multicolumn{1}{c}{ Significado } \\
\hline 1 & $\begin{array}{l}\text { Se identifican relaciones teóricas y estado de la cuestión relacionados a crisis. Se } \\
\text { reconocen señales y mecanismos de trasmisión de crisis en mercados financieros } \\
\text { de la Alianza del Pacífico. }\end{array}$ \\
\hline & $\begin{array}{l}\text { Se reconocen } 03 \text { escenarios que han (o pueden) transformar los sistemas } \\
\text { financieros internacionales: la crisis subprime, la emergencia sanitaria COVID 19 } \\
2\end{array}$ \\
& $\begin{array}{l}\text { y la pronosticada crisis de recalentamiento ruso. Se identifican señales y se } \\
\text { estructuran indicadores de precrisis y crisis. }\end{array}$ \\
\hline & $\begin{array}{l}\text { Se estiman relaciones estadísticas y econométricas en base a indicadores de } \\
\text { crecimiento económico y variables de control en escenarios de crisis financieras } \\
\text { (Ver tabla 3 y Anexo 1) }\end{array}$ \\
\hline & $\begin{array}{l}\text { Se analiza, a la luz de la evidencia y aportes teóricos la interrelación entre la } \\
\text { actividad económica de la Alianza del Pacífico frente a factores que determinan } \\
\text { su comportamiento. }\end{array}$ \\
\hline
\end{tabular}

\section{Elaboración propia}

Las características de los países de la Alianza del Pacífico, como economías emergentes se muestran en la Tabla 2.

Tabla 2: Señales e indicadores recurrentes en escenarios de crisis.

Características y muestra de países emergentes (Alianza del Pacífico)

- Países con potencial de crecimiento económico.

- Muestran condiciones de estabilidad financiera.

- Cumplen estándares de ingresos per cápita, PIB.

- Implementan políticas de impulso crediticio.

- Poseen sistemas cambiarios flexibles.

- Son receptoras de Inversión Extranjera directa: IED

- Son economías de significativa apertura comercial.

- Perciben flujos de deuda.

- Tienen escenarios de riesgo e incertidumbre.

- Pese a déficit público procuran disciplina fiscal

\section{Elaboración propia}

La Tabla 3, presenta los escenarios pertinentes de crisis globales que han sido considerados para esta investigación. 
Tabla 3: Escenarios de crisis

\begin{tabular}{ll}
\hline Escenario & Significado \\
\hline Reciente & Crisis suprime \\
\hline Presente & Emergencia sanitaria (COVID 19) \\
\hline Futuro & Recalentamiento, inflación, ambiental. \\
\hline
\end{tabular}

\section{Elaboración propia}

\section{Se explora el siguiente modelo panel:}

$$
y_{i t}=\beta_{0}+\sum_{k=1}^{K} \beta_{k} x_{k i t}+u_{i t}
$$

\section{En donde:}

- yit: Crecimiento económico de las economías de la AP, entendida como receptora de los efectos finales de choques.

- $\beta_{\mathrm{k}}$ : Coeficientes de impacto de los factores inductores estandarizados para países de la AP y para cada integrante de la misma.

- $\mathbf{X}_{\text {kit: }}$ Vector de las “ $k$ " variables inductoras de crisis, para cada país " $i$ " durante " $t$ " períodos (anuales); en este caso, para un total de 56 observaciones para el período 2007 -2020 .

- i: $1 \ldots 4$ (1=Perú, 2=Colombia, 3=Chile, 4=México).

Apostar por una modelación de panel bajo estas condiciones técnicas y estadísticas, no sólo es arriesgado, sino también insuficiente para analizar un fenómeno económico como el referido a los inductores de crisis, principalmente por el número de datos del panel. No obstante, en este estudio se toma sólo como una primera exploración para reconocer los inductores de crisis más recurrentes aún en condiciones de limitada significancia estadística. Esto quiere decir que, estamos ante un modelo de reducidas observaciones, con limitaciones conocidas a priori conocidas, pero, debe ser considerado como primera exploración para conocer los canales de los choques al interior de las economías.

Las estimaciones fueron evaluadas por MCO tradicional, efectos fijos y variables; y corregidas bajo rigurosidad econométrica. Es importante señalar que, modelos más amplios; por ejemplo, macro paneles o primeras diferencias y, con acceso a grandes bases de datos, podrían reforzar estas primeras evidencias. Estos resultados no se conducen por los resultados de esta modelación. Hay que tomar en cuenta que se aborda sólo cuatro 
países -AP- y para datos anuales (2007-2020). Si bien ello, no garantiza resultados estadísticamente confiables si genera nuevas hipótesis y pistas de investigación.

\section{RESULTADOS}

A continuación, se presentan los principales resultados de investigación.

\subsection{Explicaciones teóricas de crisis}

Un primer hallazgo es que, las crisis no tienen teoría universal. Por ser eventos contrarios a lo esperado, en base a señales, se puede lograr cierto grado de certeza respecto a su ocurrencia, pero no de su grado y nivel de predictibilidad en cuanto a sus efectos.

La Tabla 4, sintetiza estos aportes. Se encuentra que, las teorías de crisis son de caos; y, los agentes pueden confundir la naturaleza de los choques, creyendo que son transitorios, los que son permanentes; o lo contrario. En cualquier caso, afecta a las expectativas, genera incertidumbre; y los agentes -incluido el estado- podrían reaccionar retroalimentando los efectos del choque.

Tabla 4: Principales teorías, enfoques y explicaciones de crisis mundiales y financieras

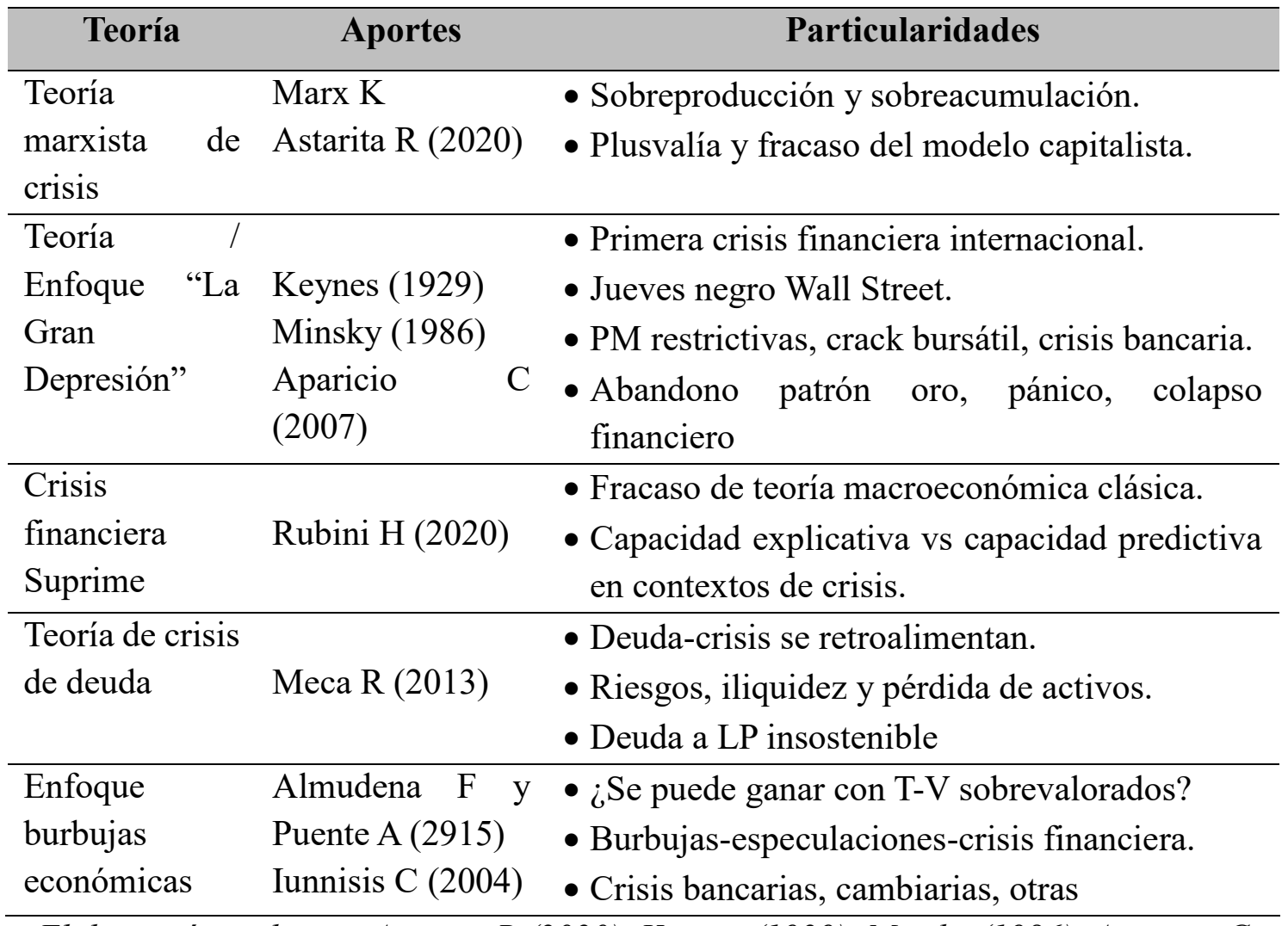

Elaboración en base a Astarita R (2020), Keynes (1929), Minsky (1986), Aparicio C

(2007), Rubini (2020), Meca R (2013), Iunnisis (2013) 
Las teorías y/o enfoques de crisis identificadas y mostradas en la tabla 4 no son todas las que ofrece la literatura, pero si las más importantes y las que comparadas muestran similitudes y diferencias que deben ser tomadas en cuenta. A partir de ellas, se encuentra que, cada crisis incorpora nuevas formas y naturaleza de choques, pero siempre terminan expandiéndose en los mercados reales y financieros de los países.

\subsection{Señales en crisis internacionales}

El siguiente resultado (Tabla 5), reafirma lo señalado. Estas tablas; además de mostrar las principales señales que están recurrentemente presentes en escenarios de crisis, permite identificar señales e indicadores que se muestran como causas, señales, inductores o efectos de los choques. Como se aprecia, incluyendo el escenario actual y futuro al 2023, en los choques internacionales siempre hay deterioro de los mercados reales y financieros. Tipo de cambio, tasas de interés, créditos internos, apertura, IED, deuda y contracción de la actividad económica son recurrentes en presencia de choques; algunos como causas, otros como consecuencias.

\section{Tabla 5: Señales de crisis financieras mundiales}

\begin{tabular}{|c|c|c|}
\hline $\begin{array}{c}\text { Crisis internacionales: causas y } \\
\text { efectos }\end{array}$ & Reacciones y respuestas de países & $\begin{array}{l}\text { Señales e indicadores } \\
\text { relevantes }\end{array}$ \\
\hline $\begin{array}{l}\text { - } 1^{\circ} \text { crisis mercados } \\
\text { emergentes: } 1890-91 . \\
\text { - Europa y EU: aumenta oferta } \\
\text { capitales-crédito } \\
\text { - Riesgo de quiebra-pánico } \\
\text { bancario }\end{array}$ & $\begin{array}{l}\text { - Burbujas bursátiles e } \\
\text { inmobiliarias. } \\
\text { - En AL afectó: Chile Argentina, } \\
\text { Brasil, Uruguay. } \\
\text { - Planes de Rescate. }\end{array}$ & $\begin{array}{l}\text { - Crisis monetaria } \\
\text { - Crisis de deuda } \\
\text { - Crisis bancaria. } \\
\text { - Caen precios de activos } \\
\text { financiero }\end{array}$ \\
\hline $\begin{array}{l}\text { - Crisis 1907:EU } \\
\text { - Pánico Bancos: crédito } \\
\text { - Expansión de economía mundial } \\
\text { - Desplome Wall Street }\end{array}$ & $\begin{array}{l}\text { - Burbujas bursátiles e } \\
\text { inmobiliarias. } \\
\text { - AL: Deterioro de expectativas } \\
\text { - Planes de Rescate. }\end{array}$ & $\begin{array}{l}\text { - Crisis bursátil } \\
\text { - Crisis de deuda } \\
\text { - Desplome créditos }\end{array}$ \\
\hline $\begin{array}{l}\text { - Crisis 1914: Primera Guerra } \\
\text { Mundial } \\
\text { - Crisis financiera y monetaria } \\
\text { nivel internacional. } \\
\text { - Pánico (agentes económicos) }\end{array}$ & $\begin{array}{l}\text { - Suspensión de bolsas (mundo). } \\
\text { - AL: Paraliza bolsas } \\
\text { - Convertibilidad oro: suspendida } \\
\text { - Planes rescate: No. } \\
\text { - Acción postguerra }\end{array}$ & $\begin{array}{l}\text { - Crisis en sector real } \\
\text { (contracción PBI, empleo, } \\
\text { etc). } \\
\text { - Crisis bursátil } \\
\text { - Déficit fiscal }\end{array}$ \\
\hline $\begin{array}{l}\text { - 1929-30: Gran Depresión } \\
\text { - Lucha por hegemonía mundial. } \\
\text { - Desequilibrios mundiales: } \\
\text { Hiperinflación Alemana, } \\
\text { excesiva confianza de } \\
\text { inversionistas, caída Wall Street } \\
\text { (jueves negro: 24/10). } \\
\text { - Salida de capitales (de A.L). }\end{array}$ & $\begin{array}{l}\text { - Derrumbe de bolsas: mundo. } \\
\text { - Quiebra de banca. } \\
\text { - Efectos reales: consumo, } \\
\text { deflación. } \\
\text { - AL: mantiene patrón oro, } \\
\text { reducción Xs: Chile-Perú. } \\
\text { - Default en deuda } \\
\text { - Intervención estatal: New Deal } \\
\text { - Regulación financ } \\
\text { - Renegociar deuda. }\end{array}$ & 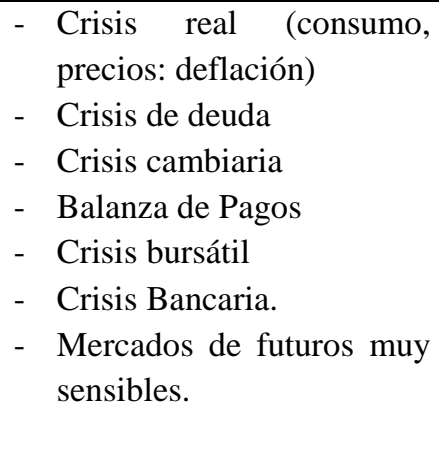 \\
\hline
\end{tabular}




\begin{tabular}{|c|c|c|}
\hline $\begin{array}{l}\text { - Crisis suprime: } 2007-2008 \\
\text { - Aparecen burbujas e ilusiones } \\
\text { financieras, malas prácticas, } \\
\text { estafas y efectos perversos. } \\
\text { - Recesión, endeudamientos, } \\
\text { inestabilidad de precios, } \\
\text { vulnerabilidades en tipo cambio e } \\
\text { interés. }\end{array}$ & $\begin{array}{l}\text { - Burbujas inmobiliaria y tecnológica } \\
\text { - Recesión, inflación y expectativas. } \\
\text { - Falta credibilidad. } \\
\text { - AL: Mercados: reales (contraídos: PBI, } \\
\text { desempleo, TC); financieros } \\
\text { (riesgosos); bursátil (sensibles). } \\
\text { - AL: Chile, México, Brasil, Argentina. }\end{array}$ & $\begin{array}{l}\text { - Crisis real (consumo, } \\
\text { precios: deflación) } \\
\text { - } \text { Crisis de deuda } \\
\text { - } \text { Crisis cambiaria } \\
\text { - } \text { Balanza de Pagos } \\
\text { - } \quad \text { Crisis bursátil } \\
\text { - } \quad \text { Crisis Bancaria. }\end{array}$ \\
\hline $\begin{array}{l}\text { - 2020: Crisis emergencia sanitaria } \\
\text { (COVID 19). } \\
\text { - Contracción mundial y de AL. } \\
\text { - Mercados financieros afectado } \\
\text { - Mercados de derivados altamente } \\
\text { sensibles } \\
\text { - Traslado a ámbito doméstico }\end{array}$ & $\begin{array}{l}\text { - Contracción del PBI, PEAO, RIN. } \\
\text { - Mercados derivados y financieros } \\
\text { temerosos: riesgo, incertidumbre. } \\
\text { - Desarrollo desmesurado de negocios } \\
\text { especulativos (derivados, opciones, } \\
\text { futuros) } \\
\text { - Déficit fiscal }\end{array}$ & $\begin{array}{l}\text { - Crisis real (consumo, } \\
\text { precios: deflación) } \\
\text { - } \text { Crisis cambiaria } \\
\text { - } \text { Balanza de Pagos } \\
\text { - } \text { Crisis bancaria. } \\
\text { - } \text { Ruido político } \\
\text { - } \quad \text { Incertidumbre }\end{array}$ \\
\hline $\begin{array}{l}\text { - 2023: Escenario posible según } \\
\text { Banco Ruso y Sputnik (2021): } \\
\text { - Riesgo de guerra. Desarrollo de } \\
\text { los mercados de futuros (con } \\
\text { fuerte especulación por nuevos } \\
\text { futuros: aire, tecnología, } \\
\text { conocimiento, salud (nuevos } \\
\text { virus, cambio climático) }\end{array}$ & $\begin{array}{l}\text { - Recalentamiento de la economía } \\
\text { mundial. } \\
\text { - Crisis de alcance mundial. } \\
\text { - Insuficiencia de respuestas macro } \\
\text { prudenciales. } \\
\text { - Sobre endeudamiento. } \\
\text { - Crisis moral } \\
\text { - Tratados de paz no creíbles. } \\
\text { - Pánico bancario. }\end{array}$ & $\begin{array}{l}\text { - Recesión, inflación, } \\
\text { - Deterioro mercados } \\
\text { financieros, deuda y } \\
\text { contracción PBI } \\
\text { mundial. } \\
\text { - Déficit de BP. } \\
\text { - Crisis mercados } \\
\text { financieros } \\
\text { - Pobreza, exclusión, etc. }\end{array}$ \\
\hline
\end{tabular}

En base a este hallazgo se propone un conjunto de inductores -posibles predictores- de escenarios de choque (ver tabla 6). Se seleccionaron por su recurrencia y, por su capacidad transmisora al interior de la Alianza del Pacífico.

\section{Tabla 6: Propuesta de indicadores inductores de choques}

\begin{tabular}{|c|c|}
\hline Indicador & Relevancia \\
\hline $\begin{array}{l}\text { Sector real } \\
\text { - Crecimiento económico } \\
\text { - Inflación } \\
\text { - Ahorro/PBI }\end{array}$ & $\begin{array}{l}\text { Reflejan efectos finales de los choques; salvo en los casos de } \\
\text { choques de oferta (permanentes o transitorios). Tardan en } \\
\text { expresarse. }\end{array}$ \\
\hline $\begin{array}{l}\text { Sector financiero } \\
\text { - Tasa interés (real y depósitos) } \\
\text { - Crédito interno privado } \\
\text { - Deuda Pública } \\
\text { - Inversión Extranjera: IED }\end{array}$ & $\begin{array}{l}\text { Reflejan desequilibrios. Son transmisores de crisis y, se } \\
\text { manifiestan antes-durante y después del choque. Se retroalimentan } \\
\text { y derivan en crisis financieras internas. Requieren respuesta rápida }\end{array}$ \\
\hline $\begin{array}{l}\text { Otros } \\
\text { - Apertura Comercial } \\
\text { - Riesgo país } \\
\text { - Tipo de cambio } \\
\text { - Índice de Temor (VIX) }\end{array}$ & $\begin{array}{l}\text { Interactúan de manera simultánea. Se manifiestan antes y durante } \\
\text { un choque; y suelen dejar efectos post crisis que implican recesión, } \\
\text { macro prudencia y disciplina fiscal. }\end{array}$ \\
\hline
\end{tabular}
Elaboración en base a Marichal (2010), Lanteri (2007), Ortiz E, et.al; A, Sosa M (2021),

Janabi M, (2021), otros 


\subsection{Comportamiento emergente de la Alianza del Pacífico: 2007-2020}

En base a la estructuración de datos para el período 2007-2020 (tabla 7), se encuentra que los países de la Alianza del Pacífico se formaron en el año 2011, pero cuando sucede la crisis suprime, sus integrantes mostraban similitudes por lo que configuran en escenario comparable con la referida crisis.

Tabla 7: Crecimiento mundial, AL y países AP: 2007-2020.

\begin{tabular}{lcccccc}
\hline Años & Mundo & A.L & Perú & Colombia & Chile & México \\
\hline 2007 & 4.4 & 5.5 & 8.5 & 6.7 & 4.9 & 2.3 \\
2008 & 1.9 & 3.9 & 9.1 & 3.3 & 3.5 & 1.1 \\
2009 & -1.6 & -1.9 & 1.1 & 1.1 & -1.6 & -5.3 \\
2010 & 4.3 & 5.8 & 8.3 & 4.5 & 5.8 & 5.1 \\
2011 & 3.1 & 4.4 & 6.3 & 6.9 & 6.1 & 3.7 \\
2012 & 2.5 & 2.8 & 6.1 & 3.9 & 5.3 & 3.6 \\
2013 & 2.7 & 2.8 & 5.9 & 5.1 & 4.0 & 1.4 \\
2014 & 2.9 & 1.0 & 2.4 & 4.5 & 1.8 & 2.8 \\
2015 & 2.9 & 0.1 & 3.3 & 3.0 & 2.3 & 3.3 \\
2016 & 2.6 & -0.4 & 4.0 & 2.1 & 1.7 & 2.6 \\
2017 & 3.0 & 1.8 & 2.5 & 1.4 & 1.2 & 2.1 \\
2018 & 3.3 & 1.8 & 4.0 & 2.6 & 3.7 & 2.2 \\
2019 & 2.3 & 1.0 & 2.2 & 3.3 & 0.9 & -0.1 \\
2020 & -3.6 & -6.3 & -11.1 & -6.8 & -5.8 & -8.2 \\
\hline
\end{tabular}

https://datos.bancomundial.org/indicator/NY.GDP.MKTP.KD.ZG?end=2013\&start=2013\&view=bar

Este comportamiento también puede verse en la Figura 1, según el cual, las economías de AL siguen la tendencia mundial, aunque con mayor incidencia. Ello, podría estar significando la fuerte dependencia con el resto del mundo por alto nivel de conexión comercial y financiera que se ha mostrado en el período de análisis.

Figura 1: Crecimiento mundial, AL y países AP: 2007-2020.

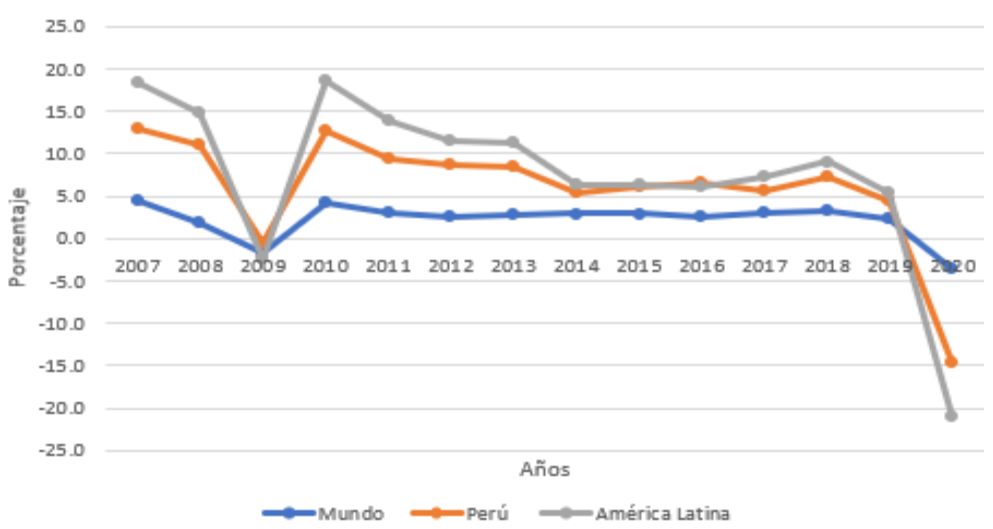

https://datos.bancomundial.org/indicator/NY.GDP.MKTP.KD.ZG? end=2013\&start=2013\&view=bar 
Tanto las tasas de interés real, como la de depósitos son reveladoras respecto a la interconexión que muestran los mercados financieros de la AP. A partir de las Figuras 2 y 3 , se observa la gran volatilidad sobre todo en Perú y Chile; mientras que Colombia y México muestran menor vulnerabilidad como resultados de sus políticas macroeconómicas.

\section{Figura 2: Tasa de interés real en países de AP: 2007-2020}

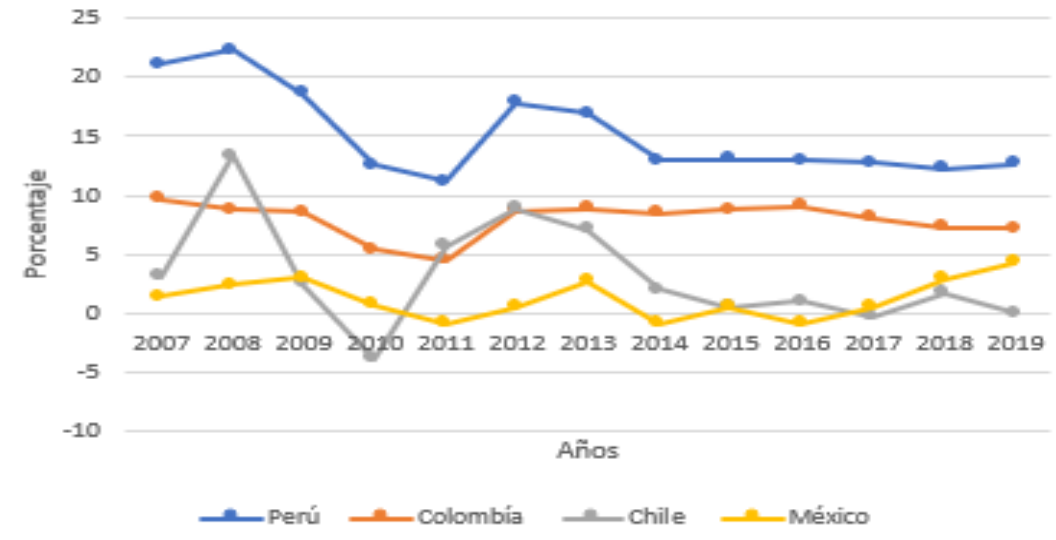

https://es.theglobaleconomy.com/rankings/Real_interest_ratel

Figura 3: Tasa de interés de depósitos en AP: 2007-2020

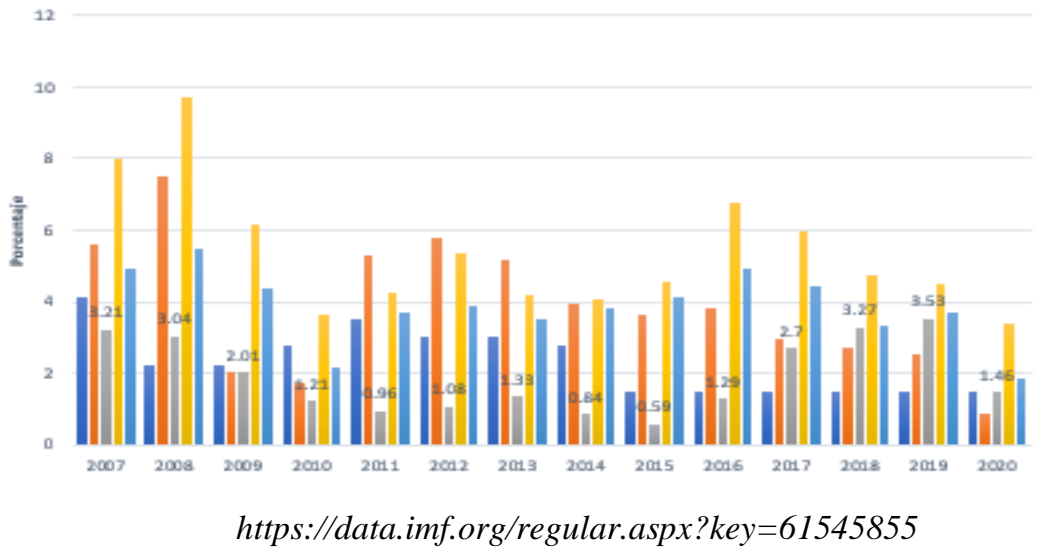

Interesante comportamiento evidencian la variable capacidad de ahorro, medido como porcentaje del PBI. La Figura 4, refleja la estabilidad de este componente de las economías de la Alianza del Pacífico, probablemente resultado de la estabilidad macroeconómica que el continente latinoamericano ha mostrado en materia económica; ello, pese a la situación socio-política también presente. 
Figura 4: Evolución del Ahorro/PBI en AP: 2007-2020

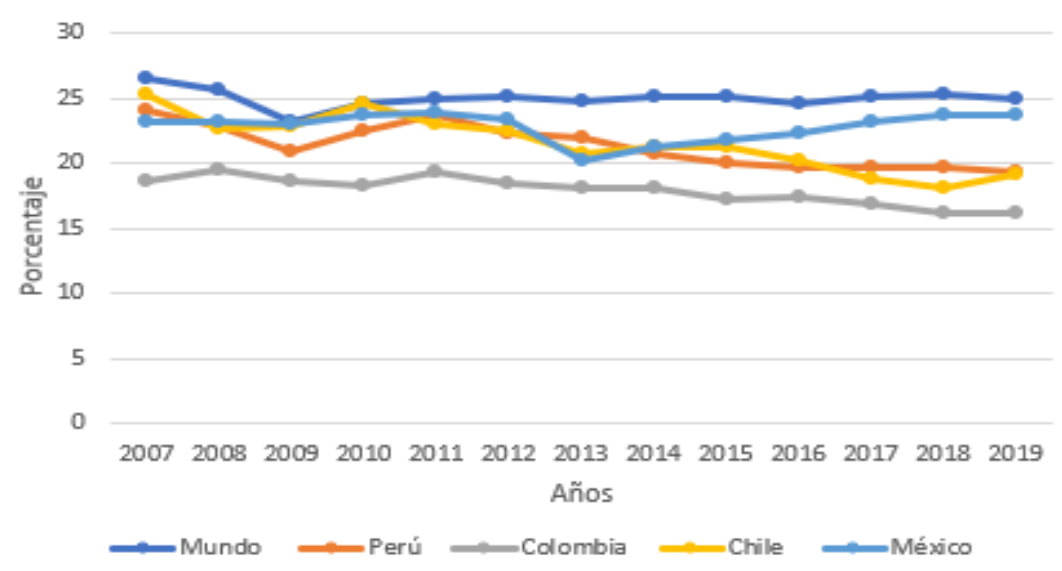

https://datos.bancomundial.org/indicator/NY.GNS.ICTR.ZS? end $=2013 \&$ start $=1961$

En relación a las variables que relacionan a estas economías emergentes de la AP, las Figuras 5 a 7, ponen de relieve particularidades respecto a la política exterior de estos países. Así por ejemplo, la apertura comercial de la AP muestra correlaciones claras y sostenidas con la apertura comercial mundial, lo que podría ser una primera gran señal de la interconexión e integración de estos mercados a la economía mundial.

Figura 5: Apertura comercial mundial, AL y AP: 2007-2020

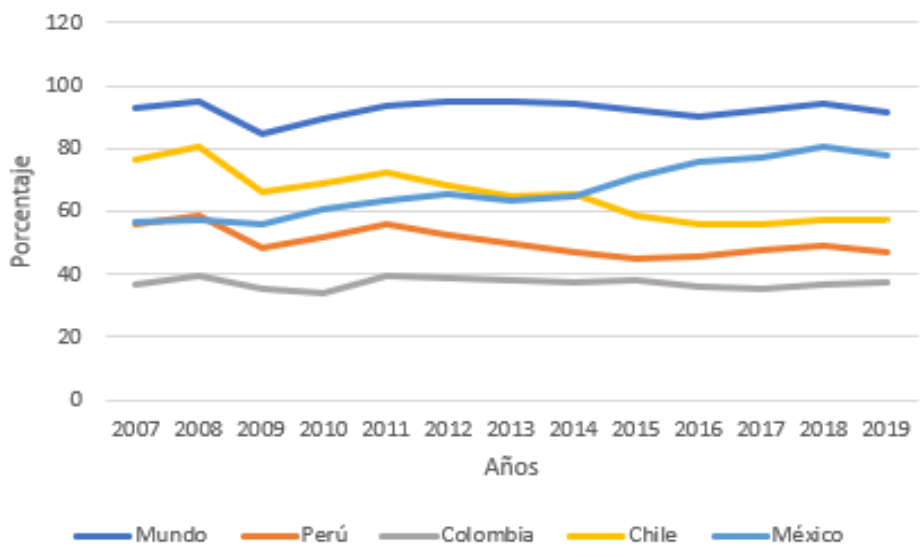

https://es.theglobaleconomy.com/rankings/trade_openness/

En este contexto, la deuda total de los países de la AP aumenta al mismo ritmo que la deuda mundial, salvo en el caso de Chile que, entre los años 2013-2015 reflejó ciertos desequilibrios aumentando su niveles de endeudamiento por encima de las tasas mostradas por el resto de países de la AP. 
Figura 6: Deuda total mundial, AL y AP: 2007-2020

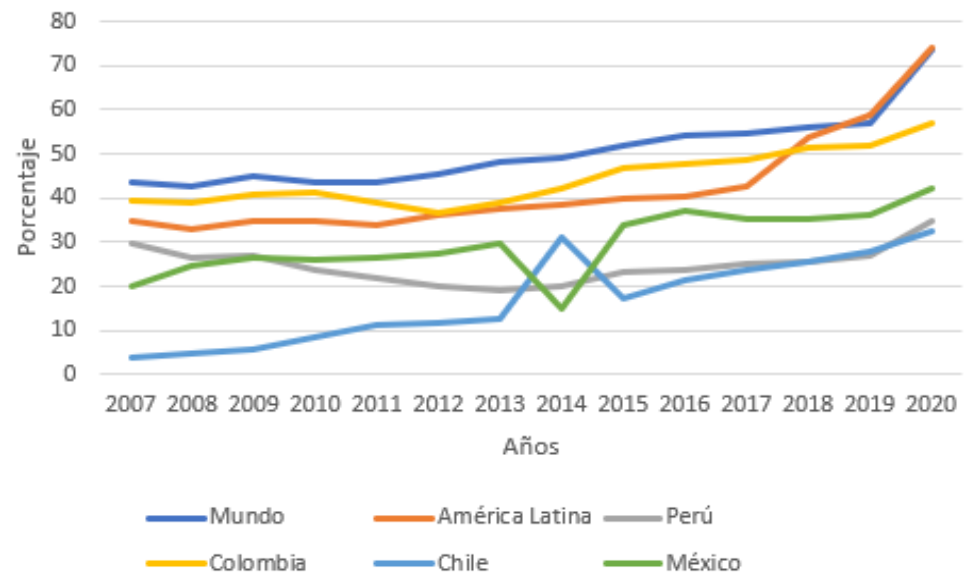

https://es.theglobaleconomy.com/rankings/Government_debt/

Por su parte, la Inversión Extranjera Directa se mostró volátil pero significativa en países como Colombia y México.

Figura 7: IED mundial, AL y países de AP: 2007-2020

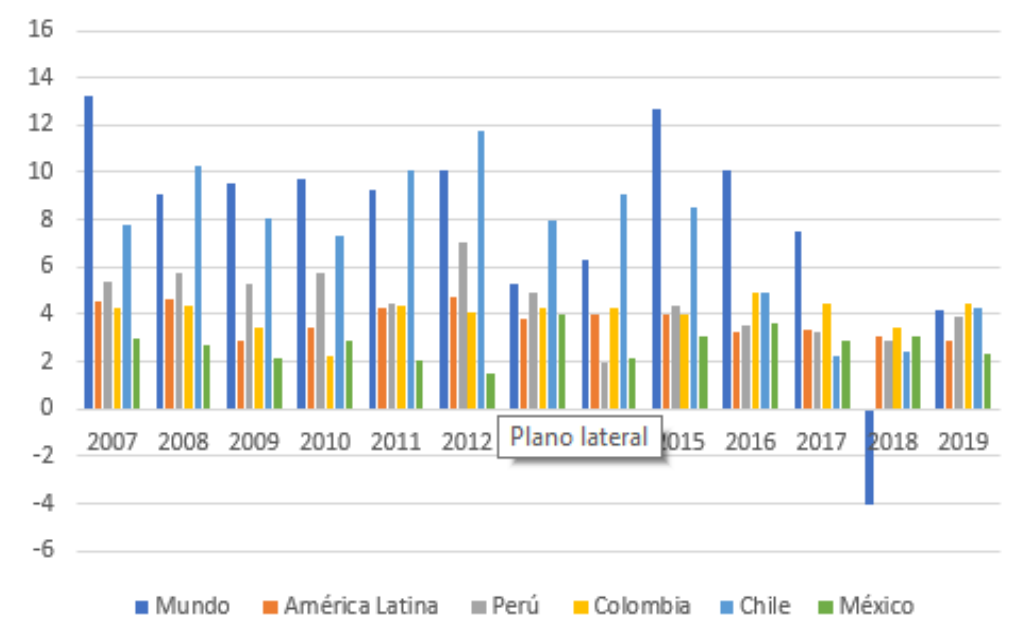

https://es.theglobaleconomy.com/rankings/Foreign_Direct_Investment/

La Figura 8, refiere a la variable inflación como diferenciadora de las políticas económicas de estabilización. Se infiere que esta variable podría ser inductora de choques, en tanto, refleja alta volatilidad, aunque a largo plazo, muestran cierta tendencia en su comportamiento y evolución. 
Figura 8: Inflación principles economías mundiales, AL y AP: 2007-2020

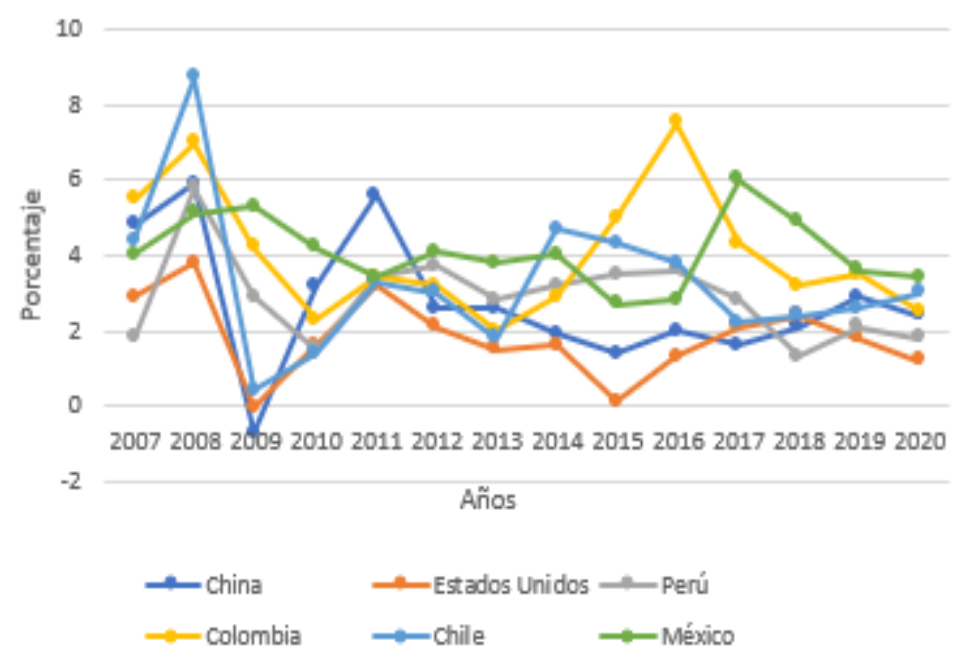

https://es.theglobaleconomy.com/rankings/Real_interest_rate/

En conclusión, a partir de este hallazgo; y después de haber evaluado las variables que posiblemente actúen como inductoras de choques; es posible reafirmar que no todas podrían serlo; algunas como el mayor endeudamiento bien podría ser efecto de los choques y vulnerabilidades.

\subsection{Inductores de choques en la Alianza del Pacífico}

El análisis de variables inductoras que canalizan los efectos de los choques al interior de las economías de la AP requiere de una primera aproximación empírica en base a un panel data aplicable a los factores comunes de la Alianza del Pacífico.

Los rezagos que dejó la crisis suprime del 2007-2008, hasta ahora visibles y, considerando el actual escenario de choque por contracción de oferta (PBI), se encontró que, bajo efectos fijos, el principal inductor de crisis sería la apertura comercial al mostrar significancia estadística y superioridad según la prueba de Hausman. Pese a la superioridad estadística de la estimación por efectos fijos, se evidencia que, al levantarse el supuesto de independencia de efectos individuales, posibilita observar efectos individuales interdependientes como realmente ocurre al interior de la Alianza del Pacífico. De esta manera, los resultados mediante efectos aleatorios consideran la interdependencia entre países; en este caso, las variables inductoras serían; además de, la apertura comercial (efectos fijos), las tasas de interés real y de depósito, y la inflación. Los resultados, con estas exploraciones al parecer serían más realistas, pero merecen una 
exploración profunda de especificación, número de variables y significancia estadística. Además, estos resultados no son concluyentes pues deben ser reestimados en contextos técnicos más completos e integrados, pero ofrecen claridad respecto a considerar empíricamente los efectos de variables invariantes, y recurrentes que facilitan el control outliers, sin tener que recurrir a variables dummy.

\section{DISCUSIÓN}

Los resultados de esta investigación nos conducen a aceptar que, en efecto, existe relación entre escenarios de crisis y el comportamiento de los mercados financieros en economías de mercados emergentes; y de manera particular en los países que conforman la Alianza del Pacífico. Estos países tienen sistemas cambiarios flexibles y están conectados con significativa apertura económica y financiera. Los mecanismos de trasmisión que se activan cuando suceden choques internacionales ocurren de diferente forma y magnitud. La forma y magnitud de sus señales y efectos dependen de las sensibilidades, que muestren los sectores real y financiero. La evidencia señala que, mercados de amplia apertura como los de la Alianza del Pacífico, son muy sensibles a escenarios de crisis, aun cuando cada país refleja determinadas diferencias. Esto ha sido recurrente en la crisis suprime, en la actual crisis por emergencia sanitaria COVID 19 y lo será en la crisis financiera mundial por recalentamiento anunciada para el año 2023.

Entonces, podemos afirmar que, entre los principales inductores de crisis resaltan la apertura comercial, pero no se puede ignorar la presencia aleatoria de otras variables inductoras, como es el caso de las tasas de interés y la inflación. Esto quiere decir, que los escenarios de crisis de carácter global; revelan sus efectos en los mercados reales alterando la estabilidad (inflación) y el comportamiento de los sistemas financieros (tasas de interés). Estos dos factores, facilitan el traslado de los choques al interior de las economías domésticas, uno hacia el sector real y el otro hacia los sistemas bancarios. En los cuatro países de la Alianza del Pacífico, se ha observado fluctuaciones en los flujos de crédito. Este impulso crediticio se correlaciona con las tasas de interés reales, y declina durante la ocurrencia choques. La evidencia señala que ello, afecta a la actividad económica en los países de la alianza; y, no existe evidencia de que alguna economía emergente se recupere sin dicho impulso. En concordancia con autores como Biggs, Mayer y Pick (2009), podemos aceptar que, ante crecimiento del crédito en escenarios de impulso crediticio, los movimientos de la tasa de crecimiento del PBI se contrarrestan, 
tanto en fases contractivas como en recuperación. Lo anterior, quiere decir que, el crecimiento está relacionado con cambios en el flujo de crédito y que, el impulso crediticio, visto como cambios en el flujo del crédito-, es particularmente relevante durante y después de recesiones.

La apertura e impulsos crediticios no son los únicos inductores de choques, pero, si los que mejor reflejan efectos en el consumo y empleo. Tanto en el escenario suprime como el de emergencia sanitaria por COVID 19, la contracción del PBI ha estado asociada a cambios contraproducentes en la liquidez, colocaciones y créditos. Estas señales son recurrentes antes, durante y después de un choque. Una forma de compensar estas fluctuaciones es controlando la variabilidad y baja del ahorro como proporción del PBI, pero también mejorando los créditos internos orientados al sector privado. Esta conexión también es recurrente en los países de la Alianza del Pacífico.

Es así como, ante la presencia de crisis se debe distinguir entre causa de choques, por ejemplo, reducción de crédito interno privado, disminución del ahorro/PBI; con señales presentes en los mecanismos de transmisión de crisis como es el caso de la IED y deuda externa total; y con los inductores de choques, que están más relacionados a las tasas de interés, la inflación, la apertura. También debe diferenciarse de los efectos finales; el cual, en este caso, lo percibe y lo muestra la contracción del PBI. En el caso de los países de la AP, conformado por México, Colombia, Chile y Perú, se encontró evidencia suficiente, respecto a que estas variables están presentes según sea el momento de las crisis. Además, aun cuando las crisis tienen diferencias y similitudes en escenarios subprime, emergencia sanitaria COVID 19 y crisis de recalentamiento.

Es así, que, reconocer las causas sirven para el diseño de políticas estructurales que vigilen el ciclo económico y su relación con los mercados reales y financieros. Las señales de crisis son útiles para anticiparse y reaccionar con rapidez y efectividad; mientras que, los inductores, sirven para anticipar choques. Una investigación como esta no concluye con un conjunto de exploraciones preliminares. Estos hallazgos apertura una serie de cuestionamientos y pistas de investigación que deben ser abordadas empíricamente para continuar entendiendo la naturaleza, inductores y efectos finales de los choques.

Queda por indagar la relación entre crisis con los mercados de capitales y el miedo natural que pueden mostrar los tenedores de activos altamente especulativos como las opciones y futuros. Así mismo, queda como desafío reconocer los escenarios de riesgo e 
incertidumbre propios de los mercados financieros, así como el riesgo-país presente en cada economía emergente de la Alianza del Pacífico. Queda pendiente, también, las conexiones de otras variables inductoras de crisis, como es el caso de la IED, la deuda y los déficits fiscales; así como, las conexiones con entornos de globalización. Por ahora, corresponde recomendar políticas agresivas de impulso crediticio, regulación financiera, apertura en condiciones de mejores ventajas competitivas para los países con economías emergentes; pero sobre todo prudencia fiscal y macro prudencia.

Es así como este estudio ha permitido reconocer las teorías de crisis financieras y sus límites en cuanto a su capacidad explicativa-predictiva. Se evidencian señales recurrentes e inductores de choques dadas las condiciones emergentes de las economías de la Alianza del Pacífico. Esto nos ha permitido comparar escenarios, reciente-presente-futuro, y lo único predecible es lo recurrente de lo impredecible.

\section{REFERENCIAS BIBLIOGRÁFICAS}

Almudena F y Puente A (2013). La burbuja inmobiliaria o efecto de la crisis: Repercusión del estallido de la burbuja inmobiliaria sobre la crisis económica. https://repositorio.comillas.edu/xmlui/bitstream/handle/11531/4637/TFG001338. pdf? sequence $=1$

Aparicio A. (2010). La política monetaria en la crisis 1929-1933: Tesis FriedmanJacobson. UNAM.

http://economia.unam.mx/profesores/aaparicio/Politica\%20Monetaria.pdf

Ardila M (2015). La Alianza del Pacífico y su importancia geoestratégica.

https://www.researchgate.net/profile/Raul-Benitez-Manaut-

2/publication/327679678_Mexico_La_trampa_diplomatica_entre_Estados_Unid os_y_America_Latina_Soft_Power_sin_Hard_Power/links/5b9e5ed8a6fdccd3cb 5c9cc4/Mexico-La-trampa-diplomatica-entre-Estados-Unidos-y-America-

Latina-Soft-Power-sin-Hard-Power.pdf\#page=245

Astarita R (2020). La Gran Depresión y la teoria marxista de las crisis.

https://laotraandalucia.org/opinion/la-gran-depresion-y-la-teoria-marxista-de-lascrisis/

Al Janabi, Mazin AM, Los derivados financieros durante la crisis de Covid-19 (Derivados financieros durante la crisis de salud de COVID-19) (2 de junio de 2021). 
SSRN: https://ssrn.com/abstract=3858999 o https://dx.doi.org/10.2139/ssrn.38 58999

Ceron, Juan A (2008). Crisis financieras internacionales, teorías explicativas y propuestas de reforma del Sistema Monetario: el caso de las subprime. Universitat de Barcelona, Facultat de Ciènces Econòmiques i Empresarials. Actas X Reunión de Economía Mundial, Barcelona.

ISSN: 1887-9489.https://e-archivo.uc3m.es/bitstream/handle/10016/2702/ceronponencia-crisis.pdf? sequence $=1 \&$ is Allowed $=\mathrm{y}$

Carrera J, Lanteri L (2007). Shocks macroeconómicos y vulnerabilidad financiera. Banco Central de la República Argentina. Ensayos Económicos, 48, 13-71. https://www.researchgate.net/publication/237272764_Shocks_macroeconómicos ___vulnerabilidad_financiera

Choy, M \& Cerna, J. (2012). Interrelación entre los mercados de derivados y el mercado de bonos soberanos del Perú y su impacto en las tasas de interés, Documentos de trabajo 2012 (21), 08 - $11 . \quad$ Recuperado de: https://www.bcrp.gob.pe/docs/Publicaciones/Documentos -deTrabajo/2012/docu mento-de-trabajo-21-2012.pdf.

Cristofoli, M. (2017). Reverse stress testing para el análisis de la estabilidad financiera española. Tesis. Doctorado en Ciencias Económicas. Universidad de Buenos Aires. $\quad$ http://bibliotecadigital.econ.uba.ar/download/tesis/15011282_CristofoliME.pdf

De la Dehesa, G. [2009]. Once fallos de mercado y de estado en la crisis financiera, Papeles de Economía Española, 2009 (122), 35-36

https://www.guillermodeladehesa.com/files/0006.1265040348.ONAW3518QQO K6741JISE4269FUIM7668.pdf

Dahrendfort R (1996). Elementos para una teoria del conflicto social. http://bibliotecadigital.udea.edu.co/handle/10495/2336

Cristofoli, M. (2017). Reverse stress testing para el análisis de la estabilidad financiera española. Tesis. Doctorado en Ciencias Económicas. Universidad de Buenos Aires. $\quad$ http://bibliotecadigital.econ.uba.ar/download/tesis/15011282_CristofoliME.pdf 
De la Dehesa, G. [2009]. Once fallos de mercado y de estado en la crisis financiera, Papeles de Economía Española, 2009 (122), 35-36 Recuperado de: https://www.guillermodela dehesa.com/files/0006.1265040348.ONAW3518QQOK6741JISE4269FUIM766 8.pdf

Dahrendfort R (1996). Elementos para una teoría del conflicto social. http://bibliotecadigital.udea.edu.co/handle/10495/2336

Jara, D. [2017]. Derivados Financieros, Descripción de mercados y Técnicas de valoración de productos derivados, Introducción a la teoría del cálculo estocástico y movimiento browniano. Quantil.

2017(2]. 08-09 Recuperado de:

https://quantil.co/wp-content/ uploads/2017/08/Derivados_agosto_2017.pdf.

Meca R (2013). De la crisis financiera a la crisis de la deuda: análisis comparativo. Cartagena.

https://repositorio.upct.es/bitstream/handle/10317/3634/tfg224.pdf?sequence=1 \&isAllowed $=\mathrm{y}$

Munchau W (2009). Una teoria para la crisis. https://www.jstor.org/stable/20647059

Martin-Fiorino, Victor; Reyes, Giovanni E. Desafíos y nuevos escenarios gerenciales como parte de la herencia del Covid-19 Revista Venezolana de Gerencia, vol. 25, núm. 90, 2020 Universidad del Zulia, Venezuela Disponible en: https://www.redalyc.org/articulo.oa?id=29063559019

Ortíz E, cabello A y Sosa M (2021). Financiarización y consumismo: $\begin{array}{lllll}\text { multipolarismos } & \text { y } & \text { crisis } & \text { covid } & 19 .\end{array}$ https://dialnet.unirioja.es/servlet/articulo?codigo=7931975

Odone CN (2000). Mercados emergentes y crisis financieras internacional. https://books.google.com/books?hl=es\&lr=\&id=fCmL_tiePI8C\&oi=fnd\&pg=PT $35 \& \mathrm{dq}=$ mercados+financieros+emergentes\&ots=DYwQU4FQgW\&sig=odMQo LQKkstgSzGYrbXFP59tFjs

Paredes, C. (2011). Los créditos del sistema bancario peruano y la crisis financiera internacional. Trujillo, Perú: Universidad Nacional de Trujillo. http://dspace.unitru.edu.pe/bitstream/handle/UNITRU/770/paredes_constante.pd $\mathrm{f}$ ? sequence $=1$ \&isAllowed $=\mathrm{y}$ 
Rubini (2010). Crisis subprime: ¿hacia una "nueva" teoría macroeconómica? Ministerio de Gobierno y Reforma del Estado | Secretaría de Tecnologías para la Gestión. https://www.santafe.gov.ar/index.php/web/content/download/82284/397971/file/ crisis_subprime.pdf

Soto Ezquivel, R. \& Correa, E. [2008]. Modelos de crisis y el uso de los instrumentos financieros derivado Problemas del Desarrollo Revista Latinoamericana de Economía, 39 (155), $14-18$.

http://www.scielo.org.mx/scielo.php?script=sci_arttext\&pid=

S03010362008000400002 .

Steinberg, F [2008]. La crisis financiera mundial: causas y respuesta política, Economía y Comercio Internacional - ARI, 2008 (26) 01-03. Recuperado de: http://biblioteca. ribei.org/id/eprint/1410/1/ARI-126-2008-E.pdf.

Torres, J. (2012). Crisis financiera. Mexico: Universidad Autónoma de México. http://conceptos.sociales.unam.mx/conceptos_final/504trabajo.pdf

Tarapuez Roa, J. (2016). Predicción de crisis financieras. Una crítica a los modelos de tercera generación.

https://repositorio.unal.edu.co/handle/unal/57785

\section{Sitios web:}

- https://quantil.co/wp-content/uploads/2017/08/Derivados_agosto_2017.pdf

- http://biblioteca.ribei.org/id/eprint/1410/1/ARI-126-2008-E.pdf https://www.guillermodeladehesa.com/files/0006.1265040348.ONAW3518QQOK67 41JISE4269FUIM7668.pdf

- https://quantil.co/wp-content/uploads/2017/08/Derivados_agosto_2017.pdf

- http://biblioteca.ribei.org/id/eprint/1410/1/ARI-126-2008-E.pdf

1. https://mundo.sputniknews.com/20210816/un-experto-pronostica-la-proximacrisis-economica-mundial-que-la-ocasionara-1115112869.html 